\title{
Review \\ Bench-to-bedside review: When is dead really dead - on the legitimacy of using neurologic criteria to determine death
}

\author{
Leslie M Whetstine
}

Graduate of Duquesne University, Health Care Ethics Center, 600 Forbes Avenue Pittsburgh, Pennsylvania 15282, USA

Corresponding author: Leslie M Whetstine, Lwhets6623@aol.com

Published: 13 March 2007

This article is online at http://ccforum.com/content/11/2/208

(c) 2007 BioMed Central Ltd
Critical Care 2007, 11:208 (doi:10.1186/cc5690)

\section{Spontaneous life versus assisted life}

The brain dead present a peculiar problem in that they are breathing corpses, which the President's Commission Report [1] attempted to explain as follows: 'When an individual's breathing and circulation lack neurologic integration, he or she is dead." The President's Commission established that artificially maintained respiration and circulation in a WBD patient are irrelevant because they are controlled by mechanical intervention rather than by the brain. They conceded that although it may look as if the patient is alive, in fact the body is not functioning in any integrated manner because it is being manipulated externally. Accordingly, they argue, 'the function and results are similar, but the source, cause, and purpose are different between those individuals with and those without functioning brains.'

We immediately encounter problems if we determine life from death based on technology if we consider that a person is not any less alive if he requires an artificial intervention. By its very definition, life-sustaining treatment serves to sustain life. Philosopher Hans Jonas [2] asks us to consider whether we would hesitate to make a dead brain function if it required an artificial intervention to do so. More likely than not, he assumes that most people would not care how the brain continued to function as long as it did so.

In this review I argue that, in its current usage, WBD is a theoretically inconsistent criterion, and that its main premise, that a functioning brain is required for integrative life, is flawed. I provide evidence that the bodies of WBD patients continue to integrate at the level of the organism as a whole and therefore fail to satisfy the classic definition of death. If ability to maintain integrated functioning is what distinguishes life from death, as the current definition of death holds, then I argue that a dead brain does not stop such functions from continuing. I conclude the review by arguing for a modification to the definition of death itself, in which the brain dead are dead, but not on the merits of the traditional biologic justification.
The Commission appeared to conflate function with the mechanism that achieves it [3]. However, there is a difference between that which sponsors the function (brain) and the function itself (for instance, respiration, circulation, and so on), and if it is the function itself that is significant and if it continues, then it ought not to matter what causes it as long as it occurs [3]. Both circulation and respiration are diffuse throughout the body, and brain failure does not stop these functions. It is true that artificial technology may be required to support them, but reliance on technology in differentiating life from death creates intractable problems. These critical

$\mathrm{HBD}=$ higher brain death; $\mathrm{ICU}=$ intensive care unit; $\mathrm{WBD}=$ whole brain death. 
functions are of the same kind that we require to cease in the WBD patient, based on the argument that they represent integration in the organism as a whole. Thus, the biologic argument for WBD espoused by the President's Commission fails as a criterion for death.

The Commission argued that the presence of integration indicates life and its absence death, but it considered integration in a WBD patient merely artifact because the brain does not direct it. Wikler [4] concludes that such a position requires the Commission to dismiss any activity in a WBD patient as 'unintegrated' unless it is directed by the brainstem. However, intensive care units (ICUs) employ apparatus that can perform many functions of the brainstem, reinforcing the view that the source of integration is irrelevant provided it can continue. Wikler argues that the Commission commits the fundamental mistake of confusing necessary with sufficient conditions. He points out that an intact brainstem in general means that a patient can breathe spontaneously, but because WBD patients are capable of respiration with assistance, this illustrates that brainstem capacity is not necessary.

\section{Integration or artifact}

The President's Commission defines integration as brain function that manifests as physiologic homeostasis [5]. Following this definition, then, WBD patients should not be able to exhibit homeostatic control. It is clear, however, that some WBD patients will continue to regulate free water homeostasis through arginine vasopressin, which does not preclude a determination of WBD. Truog and Fackler [5] argue that this is more physiologically integrative than brainstem reflexes such as pupillary constriction, which must be absent in WBD patients. Thus, using the definition endorsed by the President's Commission, some WBD patients will continue to integrate and do not meet the requirements of the classic definition of death.

Briefly, some of the integrative functions of the organism as a whole that are not controlled by the brain include homeostasis, energy balance, wound healing, infection fighting, and gestation of a fetus [6]. These are not characteristics of the dead; they are not reflexes but rather are evidence of a body that is integrated at the level of the organism as a whole. What is more disturbing is that these functions can occur in patients who have passed a WBD protocol because they are not tested when a determination of death is made [6]. WBD criteria only test the irreversible cessation of a portion of intracranial functions. For instance, the pituitary gland, cardiovascular tone, and thermoregulation generally are not tested [7].

Halevy and Brody [8] identify two areas of persistent functioning in some WBD patients that are critically integrating: neurohormonal regulation, and brain stem functioning. Furthermore, they argue that cortical function is present in some WBD patients, and although this is not required for organic integration, there is general agreement that declaring someone dead with higher brain functions intact is unacceptable.

Continued hypothalamic function, in particular, is troubling for many critics of WBD. When the brain is able to secrete antidiuretic hormones, it can prevent the development of central diabetes insipidus, which confirms that the hypothalamus and posterior pituitary are intact. This is important because, 'a functioning neurohormonal pathway is essential to the viability of the organism as a whole and it is a major example of the integrative role of the brain.' [6]. However, many patients who pass WBD protocols do not exhibit diabetes insipidus and retain residual neurohormonal regulation, which is readily assessable at the bedside; according to the most stringent definition of 'critical', this does not indicate mere activity but organized functioning. Halevy and Brody [8] observe that, "neurohormonal regulation is a component of the integrative role of the brain in regulating the rest of the body - the very role that is emphasized in the whole-brain definition of death.'

Perhaps the most convincing evidence that a WBD patient is not dead arises when such a patient successfully gestates a fetus to term. A 2003 article published in Critical Care Medicine [9] reviewed 10 such cases of women who passed WBD protocols and who were supported in ICUs in order to bring their fetuses to term. The longest period a WBD patient spent on life-sustaining treatment was in a woman who was 15 weeks pregnant at the time of admission and required support for 107 days. Surprisingly, the authors noted that, 'The clinical problems found in those women were similar to other long-term patients in ICU.' Clearly, this comparison with other ICU patients implies that these women were not corpses according to the traditional biologic definition of death.

It is clear from the discussion thus far that WBD patients can be maintained on life-sustaining treatment for much longer than was originally postulated, but the fact remains that such a claim was merely prognostic in nature and not a legitimate way to determine that death had already occurred [10]. Shewmon and others admit that total brain destruction is predictive of death but refer to extensive empirical evidence to prove that the organism as a whole, though disabled, is not yet dead $[10,11]$.

The fundamental problem is that WBD has been imposed upon society by appealing to an unsound biologic argument; WBD attempts to fulfill the definition of death as the permanent cessation of the integrated functioning of the organism as a whole despite overwhelming evidence to the contrary. At the conclusion of this paper it should be clear that WBD patients are not yet dead on the biologic merits of this definition. 


\section{Conclusion}

I suggest that the definition of death be amended from a purely biologic model to an ontologic definition that focuses on that which is essential to the human person, the loss of which constitutes death. In this regard, the justification for death is not argued on purely organic terms, because the body can continue to integrate despite a dead brain; furthermore, a strictly biologically oriented approach fails to capture that which distinguishes humans from other animals. An ontologic definition will rest on the agreement that the human brain possesses unique functions and capacities, which are significant to the nature of the human person to the extent that when the individual has irreversibly lost such capacities he or she is dead.

There is a difference between biologic life and human life. Cessation of the latter occurs when one has irreversibly lost the capacity for consciousness or 'personhood', despite persistence of the former. Although I endorse an ontologic definition, I do not advocate a higher brain death (HBD) criterion (sometimes referred to as neocortical death) because of the inability to quantify clinically the loss of such human properties, capacities, and functions. Therefore, revising the definition of death will not require us to discard the WBD criterion, which, as a purely practical matter, continues to work for society despite its shortcomings (although it may be supplanted by other criteria as medicine improves). As Youngner and Bartlett [12] note, the various criteria used to fulfill the definition of death are determined by the current medical and technical armamentarium, but the definition itself, at the philosophical level, will remain constant.

This philosophical shift allows the WBD criterion to be maintained in order to fulfill our new definition of death until tests for HBD gain greater specificity, because all those who meet WBD will necessarily meet HBD. In so doing we recognize not only the need for a defensible concept of death, because the current use of WBD rests on the faulty premises that a functioning brain is required for integrative life and that any functions that are not regulated by the brain are necessarily unintegrated, but also the need for clinical confidence. I began by claiming that WBD patients are not dead according to the traditional definition of death. I conclude with the assertion that WBD patients are dead if we adopt a new definition of death, one that focuses on the irreversible loss of consciousness as that which is essential to the human person, the loss of which portends death.

This article is part of a thematic series on End of life decision making edited by David Crippen.

Other articles in this series can be found online at http://ccforum.com/articles/ theme-series.asp?series $=$ CC_END

\section{Competing interests}

The author declares that they have no competing interests.

\section{Acknowledgement}

This paper is excerpted from portions of the author's doctoral dissertation [13].

\section{References}

1. President's Commission for the Study of Ethical Problems in Medicine and Biomedical and Behavioral Research: Defining Death: a Report on the Medical, Legal, and Ethical Issues in the Determination of Death. Washington, DC: Government Priniting Office; 1981.

2. Jonas $\mathrm{H}$ : Against the stream: comments on the definition and redefinition of death. In Philosophical Essays from Ancient Creed to Technological Man. Chicago, IL: University of Chicago Press; 1974:132-140.

3. Tomlinson T: The conservative use of the brain-death criterion: a critique. J Med Philos 1984, 9:377-393.

4. Wikler D: Brain death: a durable consensus? Bioethics 1993, 7:239-246.

5. Truog RD, Fackler JC: Rethinking brain death. Crit Care Med 1992, 20:1706.

6. Halevy A: Beyond brain death? J Med Philos 2001, 26:495-501.

7. Zamperetti N, Bellomo R, Defanti CA, Latronico N: Irreversible apnoeic coma 35 years later. Intensive Care Med 2004, 30: 1715-1722.

8. Halevy A, Brody B: Brain death: reconciling definitions, criteria, and tests. Ann Internal Med 1993, 119:519-525.

9. Powner D, Bernstein I: Extended somatic support for pregnant women after brain death. Crit Care Med 2003, 31:1241-1249.

10. Byrne PA, O'Reilly S, Quay PM, Salsich Jr PW: Brain death: the patient, the physician, and society. In: Beyond Brain Death. Edited by Byrne PA, Nilges RG, Potts M. The Netherlands: Kluwer Academic Publishers; 2000:21-89.

11. Shewmon DA: The brain and somatic integration: insights into the standard biological rationale for equating 'brain death' with death. J Med Phil 2001, 26:457-478.

12. Youngner SJ, Bartlett ET: Human death and high technology: the failure of the whole-brain formulations. Ann Intern Med 1983, 99:252-258.

13. Whetstine LM: An examination of the bio-philosophical literature on the definition and criteria of death; when is dead 'dead' and why some donation after cardiac death donors are not. PhD thesis. Duquesne University; 2006. 